\title{
Experimental Study on the Dynamic Behavior of Running Crack Affected by Defect Shape and Filling Material
}

\author{
Zhen Lei ${ }^{1}$ and Mingsheng Zhao ${ }^{2}$ \\ ${ }^{1}$ Institute of Mining Engineering, Guizhou Institute of Technology, Guizhou 550003, China \\ ${ }^{2}$ Poly Xinlian Blasting Engineering Group Co., Ltd., Guizhou 550026, China \\ Correspondence should be addressed to Zhen Lei; 20150635@git.edu.cn
}

Received 10 November 2019; Revised 27 January 2020; Accepted 3 February 2020; Published 13 March 2020

Academic Editor: Marco Alfano

Copyright (c) 2020 Zhen Lei and Mingsheng Zhao. This is an open access article distributed under the Creative Commons Attribution License, which permits unrestricted use, distribution, and reproduction in any medium, provided the original work is properly cited.

\begin{abstract}
Using the caustics method and the experimental system of digital laser dynamic caustics, the model experiment of drop hammer impact loading was carried out, and the effect of the defect shape (circular and rectangular) and the filling material (air, epoxy, and silicone rubber) on the propagation behavior of the running crack was investigated. The experimental results show that, under the impact loading, the running crack initiates at the end of precrack and propagates toward the defect. After the running crack connects to the defect, it accumulates energy within a certain period before initiating again at the upper edge of the defect. Subsequently, only one running crack is formed at the upper edge of the circular defect, but two running cracks are formed at the upper edge of the rectangular defect. The defect shape and the filling material have a significant effect not only on the energy accumulation time of the running crack at the defect but also on the stress intensity factor when initiating at the defect. The effect degree of the defect shape on the running crack propagation behavior is in the following order: circular defect $>$ rectangular defect, whereas the effect degree of the filling material on the running crack propagation behavior follows this order: air $>$ silicone rubber $>$ epoxy.
\end{abstract}

\section{Introduction}

Defects such as cracks and empty holes inevitably contained in the engineering structure seriously impact their strength and mechanical properties [1]. Therefore, the fracture behavior of defective structures has always been a key topic in related disciplines and has been investigated in detail, leading to many important results.

In terms of theoretical research, Wasiluk and Golos [2] established the $Z$ criterion and considered that the crack propagation direction can be determined by the minimum $Z$ factor. In order to investigate the fracture behavior under mixed-mode loading, Aliha et al. [3] theoretically analyzed the fracture characteristic of the cracked ring specimen subjected to diametral compressive loading. Zhang et al. [4] used the elastoplastic large defection model combined with the finite difference method to analyze the dynamic behavior of the free beam containing a defect. Based on the theory of linear fracture mechanics and the principle of strain energy release, $\mathrm{Hu}$ et al. [5] obtained the effect of crack on the natural frequency of the structure.

In terms of experimental research, Cortet et al. [6] found a mutually exclusive area and attractive area when the two cracks propagated in the opposite direction. Marsavina et al. [7] presented a correlation between the dynamic and static fracture toughness of polyurethane rigid foams, indicating that, for all foam densities, the dynamic fracture toughness is higher than the static toughness. Koivisto et al. [8] and Fender et al. [9] conducted further research on such findings and analyzed it from the perspective of interaction of the running crack. Yang et al. [10] used the caustics method to study the suppression or promoting effect of an empty hole on the propagation of the adjacent running crack. Yue et al. [11] studied the effect of heterogeneous inclusions on the dynamic fracture behavior of the beam. In addition, Linul et al. experimentally investigated the main mechanical 
properties of the closed-cell aluminum-alloy foams containing a lot of empty holes under different quasi-static loading conditions and different temperatures [12].

In terms of numerical simulation, Li et al. [13] used AUTODYN program to study the effect of an empty hole on the propagation of the blast-induced crack. The finite-element results conducted by Aliha et al. [14] have shown that the edge crack triangular specimen that contained a precrack is able to provide pure mode I, pure mode II, and any mixedmode loading conditions between the two. Zhou et al. [15] studied the impact fracture characteristics of brittle material containing precracks and analyzed the stress characteristics and the propagation path of crack. Wu and Wong [16] used the numerical manifold method to study the effect of microcracks on the mechanical properties of granite under different loading rates, revealing that the dynamic strength and deformation performance of granite increased with increasing loading rate. In addition, Marsavina et al. [17] used the displacement correlation method to calculate the fracture parameters, the remesh, and fill algorithm and implemented the minimum strain energy density criteria in the Finite-Element Analysis to accurately predict the curvilinear crack propagation path. Berto and Ayatollahi [18] reanalyzed some recent experimental fracture results from Brazilian disk specimens weakened by blunt $\mathrm{V}$-notches in terms of local SED characterized by different degrees of load mixity.

In addition, the three-point bend test is a commonly used method to study the fracture behavior of materials under both static loading and dynamic loading. For polymethyl methacrylate (PMMA), Chao et al. [19] studied the mode I fracture behavior of PMMA using an edge cracked beam subjected to three-point bend loading, double cantilever beam, and compact tension specimens. They showed that the geometry and loading type of test specimen may affect noticeably the mode I fracture toughness of PMMA. Then, the circular, semicircular, and square shape specimens have been used by Ayatollahi and Aliha [20], Aliha and Ayatollahi [21], and Saghafi et al. [22], respectively, for obtaining pure mode I and mixed-mode I/II fracture toughness of PMMA.

Engineering structure has defects of various shapes, and these defects are often filled by other media, making the dynamic fracture process of the structure more complicated. Therefore, studying the effect of defect shape and filling material on the dynamic fracture behavior of structures is of great scientific and engineering significance. Based on this, in this study, we investigated the effect of defect shape and filling material on the fracture characteristics and crack propagation behavior in the PMMA specimen under dynamic impact loading by the three-point bend test.

\section{The Caustics Method and Experimental System}

2.1. Principle of the Caustics Method. The optical properties of a solid medium change under stress loading. Due to the Poisson effect, under the action of tensile stress, the thickness of the medium decreases and becomes an optically thinner medium; under the action of compressive stress, the exact opposite phenomenon is observed. Taking the mode I crack generated under tensile stress as an example, as shown in Figure 1, the stress concentration around the crack changes the optical properties of the local medium at the crack tip. The parallel incident lights are refracted at the crack tip region, resulting in no light irradiation in the corresponding region of the reference plane, thereby forming a shaded area. The shaded area of the reference plane is the caustic spot, and the boundary between the caustic spot and the surrounding bright area is the caustics.

The mapping equation of the caustics on the reference plane is expressed by

$$
\left\{\begin{array}{l}
x^{\prime}=r \cos \theta-\frac{z_{0} c d r^{-3 / 2}}{\sqrt{2 \pi}}\left(\cos \frac{3 \theta}{2} K_{\mathrm{I}}-\sin \frac{3 \theta}{2} K_{\mathrm{II}}\right), \\
y^{\prime}=r \sin \theta-\frac{z_{0} c d r^{-3 / 2}}{\sqrt{2 \pi}}\left(\sin \frac{3 \theta}{2} K_{\mathrm{I}}+\cos \frac{3 \theta}{2} K_{\mathrm{II}}\right),
\end{array}\right.
$$

where $r$ is the polar radius, $\theta$ is the polar radius, $z_{0}$ is the distance between the specimen and the reference plane as shown in Figure 1, $c$ is the optical stress constant of the specimen material, $d$ is the thickness of the specimen, $K_{\mathrm{I}}$ is the mode I stress intensity factor of the crack tip, and $K_{\mathrm{II}}$ is the mode II stress intensity factor of the crack tip.

In this study, the propagation behavior of the mode I crack was mainly studied. The caustics of the mode I crack shown in Figure 2 were obtained according to (2). The "+" in the figure indicates the light transmitted to the reference plane, and the blank area in the figure corresponds to the caustic spot.

2.2. The Experimental System. The experimental system of digital laser dynamic caustics [23] as shown in Figure 3 was established by the Institute of Modern Blasting Technology Application, China University of Mining and Technology (Beijing). The experimental system mainly comprises a laser device, a beam expander, a field lens, a loading device, and a high-speed camera. Experiments performed by the loading device were completely recorded using the high-speed camera.

\section{Experimental Design and Parameter Calculation}

3.1. Experimental Design. The polymethyl methacrylate (PMMA) was used as the specimen material, with a size of $220 \times 50 \times 5 \mathrm{~mm}^{3}$. PMMA is a typical brittle material, and its main dynamic mechanical parameters are listed in Table 1.

In order to compare the effects of the circular defect and rectangular defect on the running crack propagation behavior caused by the drop hammer impact, two experimental groups, as shown in Figure 4, were set up, namely, the circular defect group and the rectangular defect group. Relevant size parameters are also shown in the figure. In addition, in order to study the effect of filling material property on the running crack propagation behavior, the 


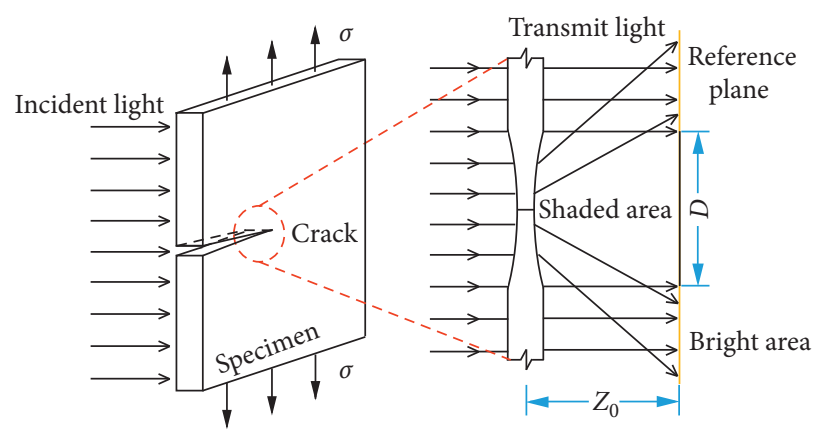

Figure 1: Imaging diagram of the caustics.

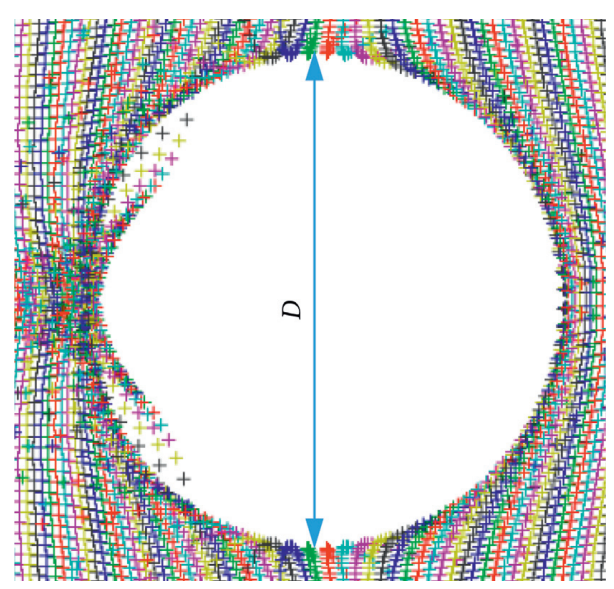

Figure 2: The morphology of mode I caustics.

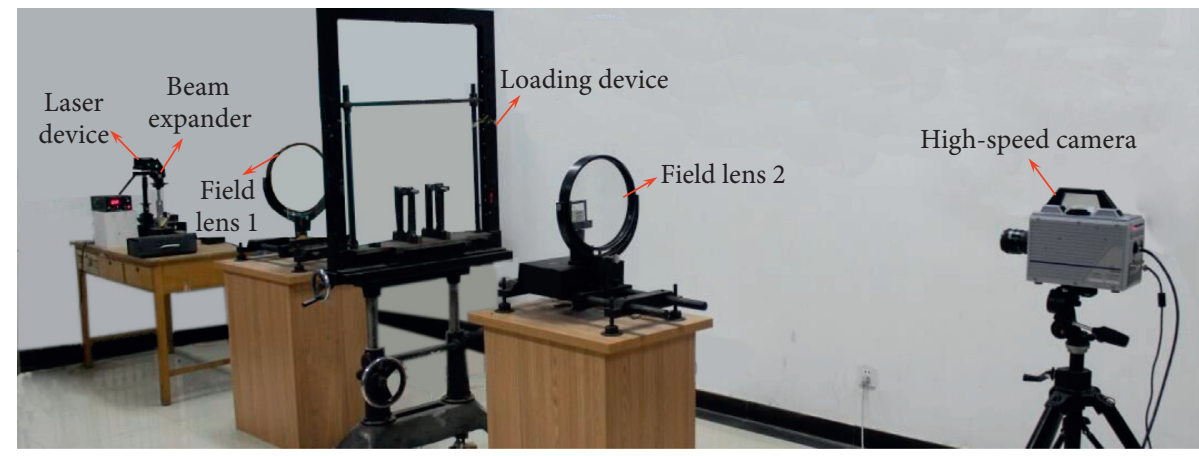

Figure 3: The experimental system of digital laser dynamic caustics.

TABle 1: Dynamic mechanical parameters of PMMA.

\begin{tabular}{lccc}
\hline P-wave velocity $(\mathrm{m} / \mathrm{s})$ & S-wave velocity $(\mathrm{m} / \mathrm{s})$ & Dynamic elastic modulus $(\mathrm{GPa})$ & Dynamic Poisson ratio \\
\hline 2320 & 1260 & 6.1 & 0.31 \\
\hline
\end{tabular}

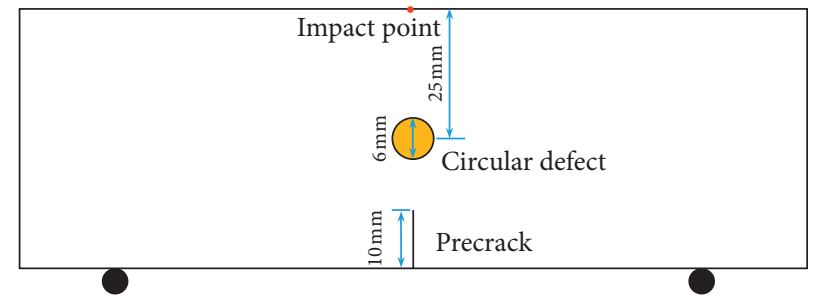

(a)

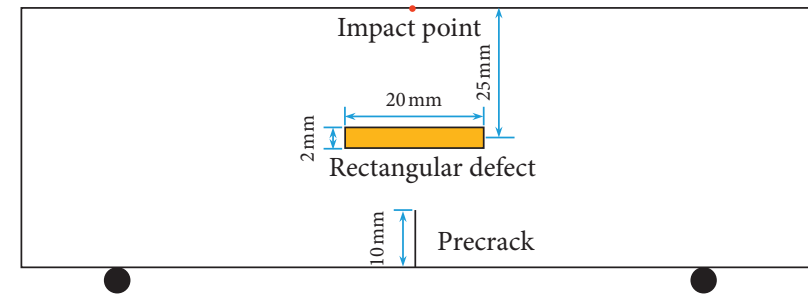

(b)

FIgURE 4: Diagram of the specimen containing a defect. (a) Circular defect group. (b) Rectangular defect group. 
defects of specimens were filled with different materials. In the circular defect group, air, epoxy (brittle material), and silicone rubber (flexible material) were used as the filling material in the defect and were referred as specimens $C_{\mathrm{a}}, C_{\mathrm{e}}$, and $C_{s}$, respectively. Similarly in the rectangular defect group, air, epoxy (brittle material), and silicone rubber (flexible material) were used as the filling material in the defect and were referred specimens $T_{\mathrm{a}}, T_{\mathrm{e}}$, and $T_{\mathrm{s}}$, respectively.

In the experiment, the mass of the drop hammer is $1 \mathrm{~kg}$, and the drop height of the drop hammer is $300 \mathrm{~mm}$, meeting the requirements of dynamic impact loading. The shooting speed of the high-speed camera was set as $210,000 \mathrm{fps}$, recording 210,000 photos/s, and the time interval between two successive photos was $4.76 \mu \mathrm{s}$.

3.2. Parameter Design. The stress intensity factor is the physical quantity that characterizes the stress field at the crack tip, and the stress intensity factor of mode I crack can be calculated as [24]

$$
K_{\mathrm{I}}=\frac{2 \sqrt{2 \pi} F(v)}{3 g^{5 / 2} z_{0} c d} D^{5 / 2},
$$

where $K_{\mathrm{I}}$ is the mode I stress intensity factor, $D$ is the diameter of the caustics at the mode I crack tip shown in Figure 2, $F(v)$ is the correlation factor associated with the crack propagation velocity, $F(v) \approx 1 ; g$ is the numerical factor, for PMMA material and is equal to $3.02, z_{0}, c$, and $d$ are the same as in (1), and in this experiment, $z_{0}=0.9 \mathrm{~m}$, $c=1.08 \times 10^{-10} \mathrm{~m}^{2} / \mathrm{N}$, and $d=5 \mathrm{~mm}$.

The center of the caustic spot is the position where the crack tip is located; therefore, the position of the crack tip at each moment can be obtained from the caustics photos taken by the high-speed camera. Since the time interval between the two successive caustics photos is extremely short $(4.76 \mu \mathrm{s})$, the average crack velocity of the two successive photos can be regarded as the instantaneous velocity of crack propagation. It is calculated as follows:

$$
v \approx \frac{\Delta s}{\Delta t}
$$

where $v$ is the instantaneous velocity of crack propagation, $\Delta s$ is the crack tip displacement difference between two successive caustics photos, and $\Delta t$ is the time interval between two successive caustics photos, $\Delta t=4.76 \mu \mathrm{s}$.

\section{Interaction Process between Running Crack and Defect}

There are three specimens of each type, and repeated experiments were performed under the same loading conditions. A preliminary analysis indicated good repeatability of the experiment. Therefore, a random specimen was selected from each type for the following analysis.

In the experiment, the initiation moment of the running crack at the end of precrack was recorded as $t=0 \mu \mathrm{s}$. According to the fracture process of the specimen under impact loading, the dynamic behavior of the running crack can be divided into three stages. Stage I: the running crack initiates at the precrack and then propagates to the lower edge of the defect. Stage II: the running crack stops propagating at the defect and accumulates energy. Stage III: the running crack initiates again at the upper edge of the defect and then propagates through the specimen. The detailed analysis of the interaction process between the running crack and defect is presented in the following sections.

4.1. Analysis of the Circular Defect Group. Figure 5 shows the caustics photos of the interaction between the running crack and circular defect of specimens $C_{\mathrm{a}}, C_{\mathrm{e}}$, and $C_{\mathrm{s}}$. In the whole process of running crack initiation and propagation, the caustics at the crack tip always exhibit a mode I morphology. In stage I, after initiation, the running crack propagates toward the circular defect and reaches the lower edge of the circular defect at $t=33.3 \mu$ s and then connects with the defect. The propagation process of the running cracks in specimens $C_{\mathrm{a}}, C_{\mathrm{e}}$, and $C_{\mathrm{s}}$ is basically the same in this stage.

In stage II, after the running crack connects with the defect, it does not initiate directly at the upper edge of the defect. For specimen $C_{a}$, Figure 5(a) shows that, at $t=366.7 \mu \mathrm{s}$, a crescent-shaped caustic spot was formed around the circular defect. The upper edge of the circular defect is subjected to tensile stress, the elastic strain energy is concentrated, and the running crack is about to initiate again at the upper edge of the circular defect. Specimens $C_{\mathrm{e}}$ and $C_{\mathrm{s}}$ also exhibit similar energy concentration characteristics in this stage, except that the energy accumulation time showed a difference. The energy accumulation times of specimens $C_{\mathrm{a}}, C_{\mathrm{e}}$, and $C_{\mathrm{s}}$ are $333.4,95.3$, and $190.5 \mu \mathrm{s}$, respectively; i.e., the air-filled energy accumulation time is the longest, followed by the silicone rubber-filled energy accumulation time, and then epoxy-filled energy accumulation time.

In stage III, the strain energy around the circular defect rapidly released, and the running crack starts to initiate at the upper edge of the circular defect. The figure shows that the caustic size of the running crack is significantly larger than that in stage I, indicating that the running crack is more difficult to initiate at the upper edge of the circular defect. Thereafter, the running crack continues to propagate toward the impact point of drop hammer until the specimen is completely fractured.

The effect of circular defect diameter and filling material stiffness on the crack velocity and dynamic stress intensity factor has been investigated using a similar research method [25]. The test results indicate that the presence of circular defect before the running crack decreases both the velocity and the stress intensity factor, which is consistent with the results obtained from the above analysis. In addition, it indicated that the circular defect with $8 \mathrm{~mm}$ in diameter shows maximum retardation.

4.2. Analysis of the Rectangular Defect Group. Figure 6 shows the caustics photos of the interaction between the running crack and rectangular defect in specimens $T_{\mathrm{a}}, T_{\mathrm{e}}$, and $T_{\mathrm{s}}$. The dynamic behavior of the running crack is different from that of the circular defect group. In stage I, after initiation, the 


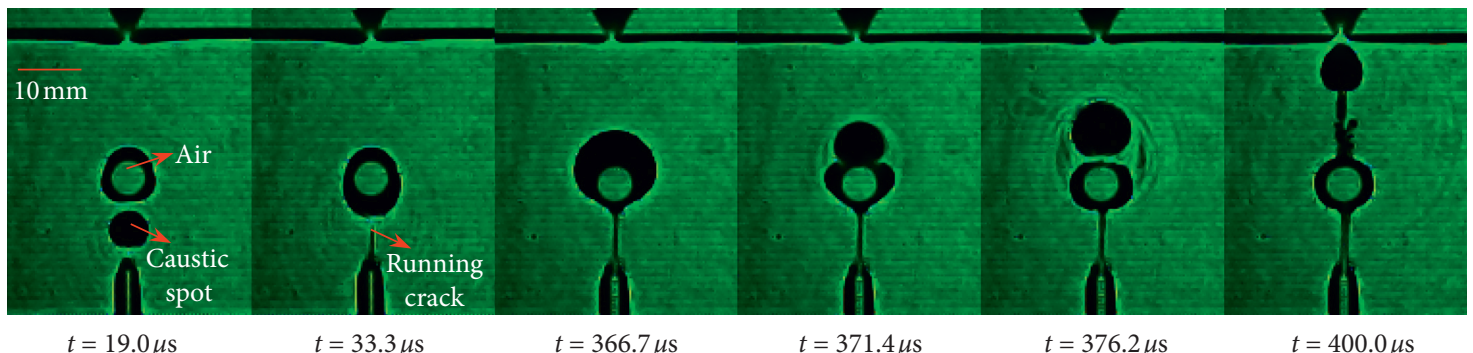

(a)
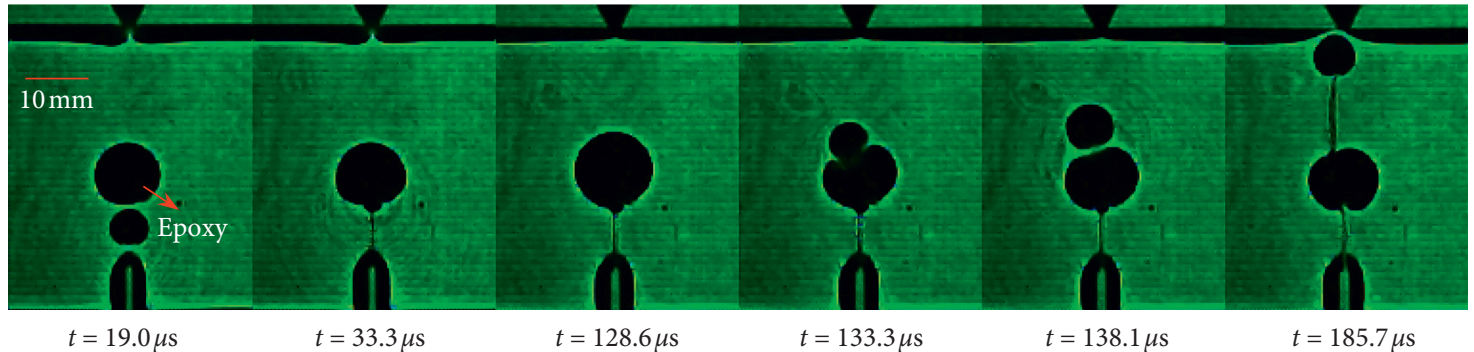

$t=133.3 \mu \mathrm{s}$

$t=138.1 \mu \mathrm{s}$

$t=185.7 \mu \mathrm{s}$

(b)

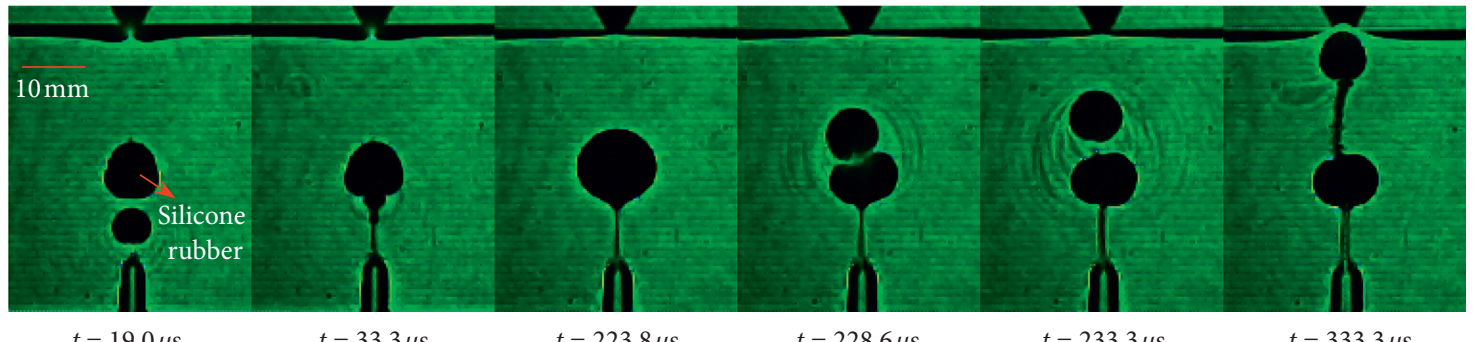

(c)

FIGURE 5: Caustics photos of the interaction between the running crack and circular defect. (a) Specimen $C_{\mathrm{a}}$. (b) specimen $C_{\mathrm{e}}$. (c) Specimen $C_{s}$.

running crack propagates toward the rectangular defect and reaches the lower edge of the circular defect at $t=42.9 \mu \mathrm{s}$ and then connects with the defect. The propagation process of the running cracks in specimens $C_{\mathrm{a}}, C_{\mathrm{e}}$, and $C_{\mathrm{s}}$ is basically the same in this stage.

In stage II, similar to the case of the circular defect group, running crack also undergoes a process of propagating stagnation and energy accumulation at the rectangular defect. For specimen $T_{\mathrm{a}}$, Figure 6(a) shows that, at $t=123.8 \mu \mathrm{s}$, the caustic spot is formed at the upper left corner and upper right corner of the rectangular defect, and running cracks are about to initiate. The energy accumulation time of the running cracks at the rectangular defect of specimen $T_{\mathrm{a}}$ is $80.9 \mu \mathrm{s}$. Similarly, the energy accumulation times of specimens $T_{\mathrm{e}}$ and $T_{\mathrm{s}}$ are 57.1 and $71.4 \mu \mathrm{s}$, respectively. The air-filled energy accumulation time is the longest, followed by the silicon rubber-filled energy accumulation time, and then the epoxy-filled energy accumulation time. Such effect of the filling material on energy accumulation time is consistent with the effect of the circular defect group. In addition, when the filling materials are the same, the energy accumulation time at the rectangular defect is relatively small compared to that at the circular defect.
In stage III, the strain energy at the upper left corner and upper right corner of the rectangular defect rapidly released, forming two running cracks, indicating that the caustic spot size of the running crack propagating from the rectangular defect is significantly smaller than that propagating from the circular defect. It shows that the running crack is easier to initiate at the rectangular defect than the circular defect.

\section{Dynamic Propagation Behavior of Running Crack}

5.1. Analysis of the Circular Defect Group. Figure 7 shows the changing curves of stress intensity factor $K_{\mathrm{I}}$ and propagation velocity $v$ of the running crack with time in the circular defect group. In stage I, when initiating at the end of the precrack, the stress intensity factor of the running cracks in specimens $C_{\mathrm{a}}, C_{\mathrm{e}}$, and $C_{\mathrm{s}}$ is $0.84,0.88$, and $0.85 \mathrm{MN} \cdot \mathrm{m}^{-3 / 2}$, respectively. After the running crack initiation, both the stress intensity factor and propagation velocity increase first and then decrease.

After the energy accumulation in stage II, the propagation process of the running crack enters stage III. In this 


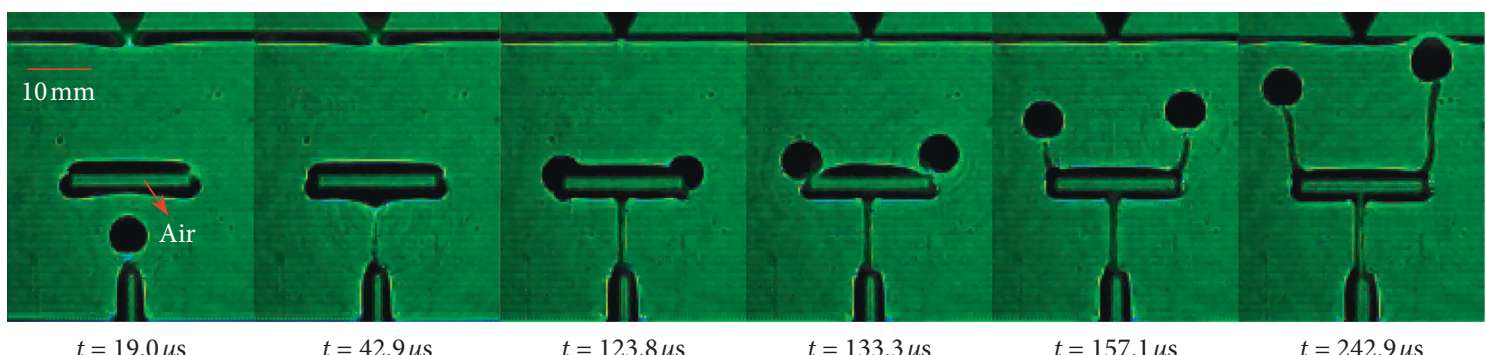

(a)

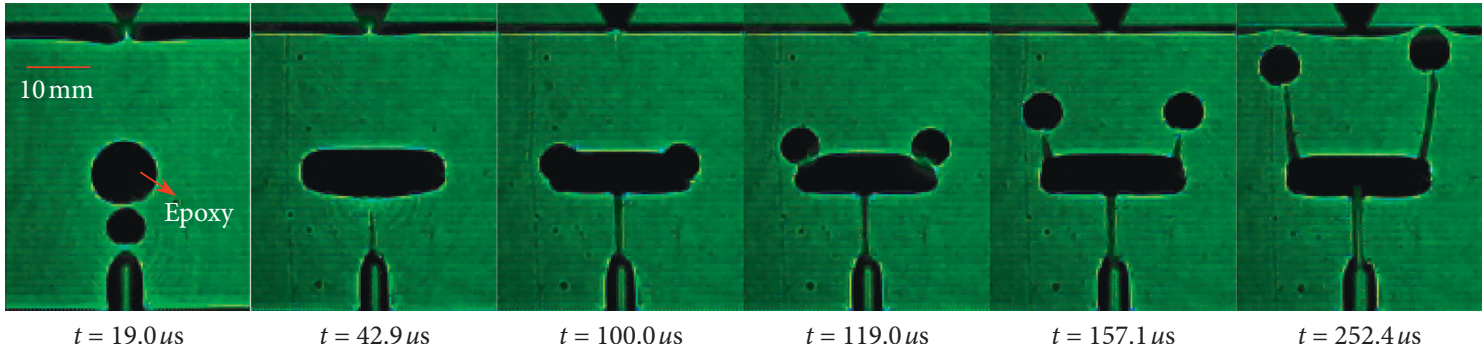

(b)

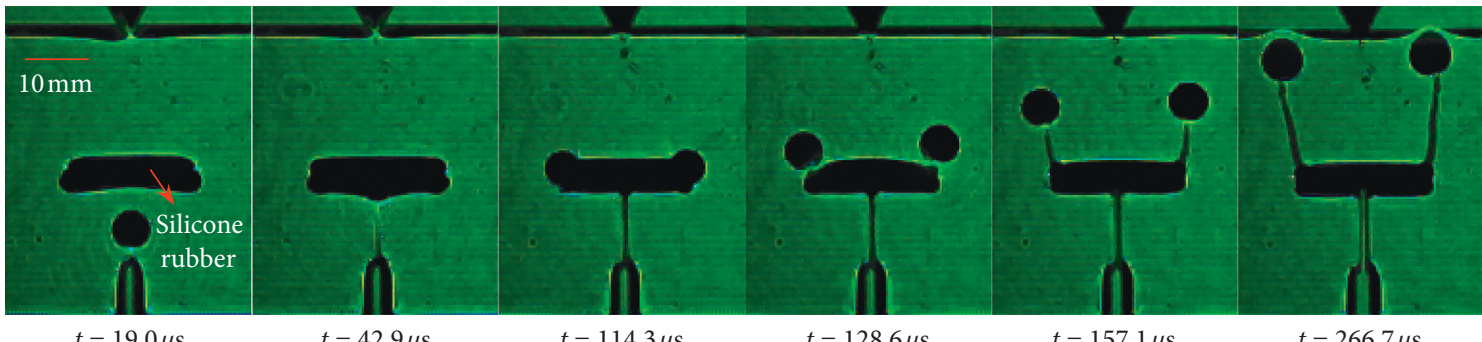

(c)

Figure 6: Caustics photos of the interaction between the running crack and rectangular defect. (a) Specimen $T_{\mathrm{a}}$. (b) Specimen $T_{\mathrm{e}}$. (c) Specimen $T_{s}$.

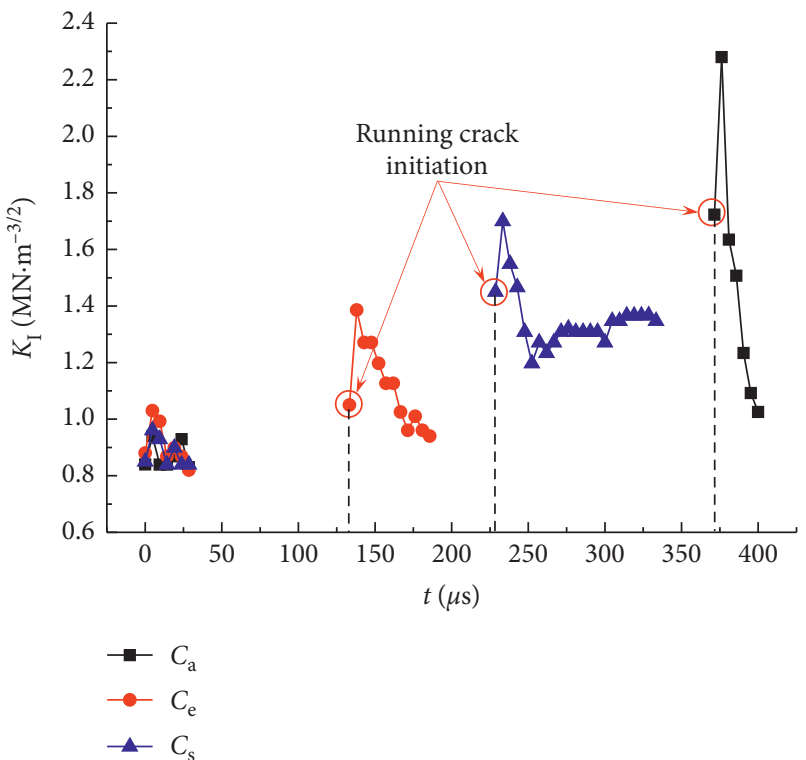

(a)

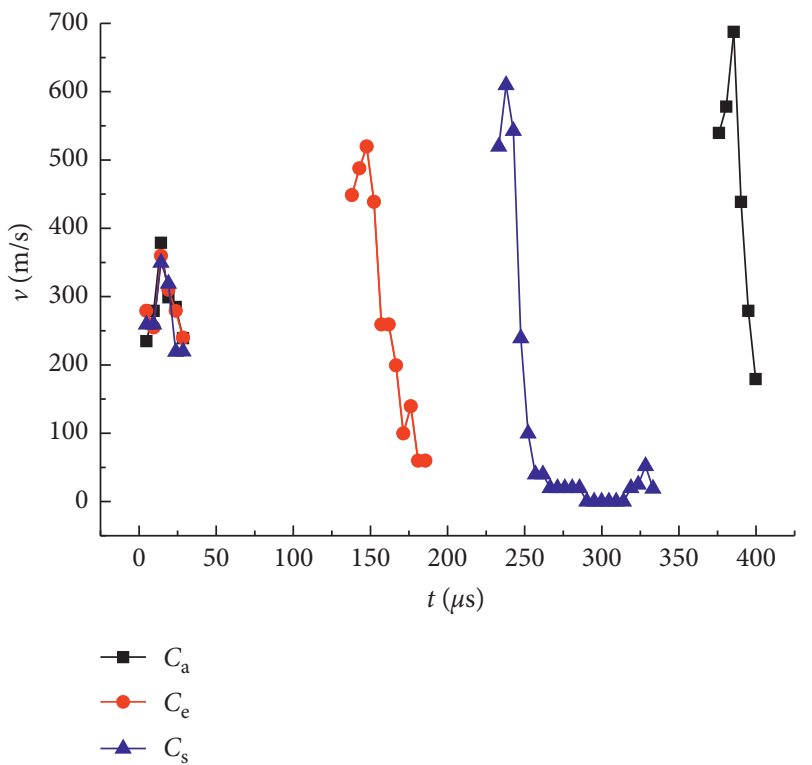

(b)

FIgURE 7: Changing curves of the dynamic mechanical parameters of the running crack with time in the circular defect group. (a) $K_{\mathrm{I}}-t$. (b) $v-t$. 
TABLE 2: The initiating time and corresponding stress intensity factor at the upper edge of the circular defect.

\begin{tabular}{lccc}
\hline Specimen & $C_{\mathrm{a}}$ & $C_{\mathrm{e}}$ & $C_{\mathrm{s}}$ \\
\hline Initiating time $(\mu \mathrm{s})$ & 371.4 & 133.3 & 228.6 \\
Stress intensity factor $\left(\mathrm{MN} \cdot \mathrm{m}^{-3 / 2}\right)$ & 1.72 & 1.05 & 1.45 \\
\hline
\end{tabular}

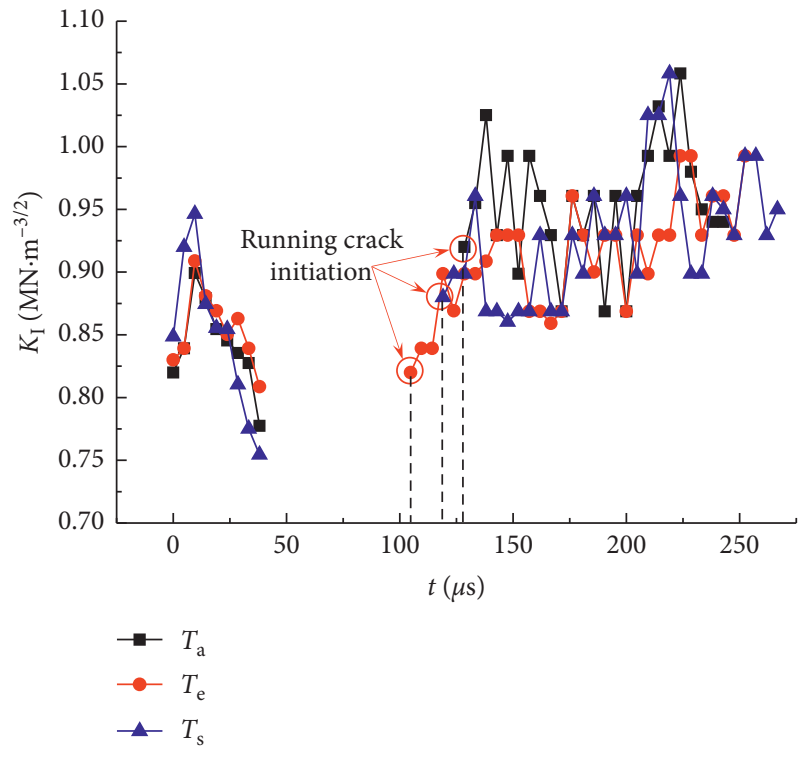

(a)

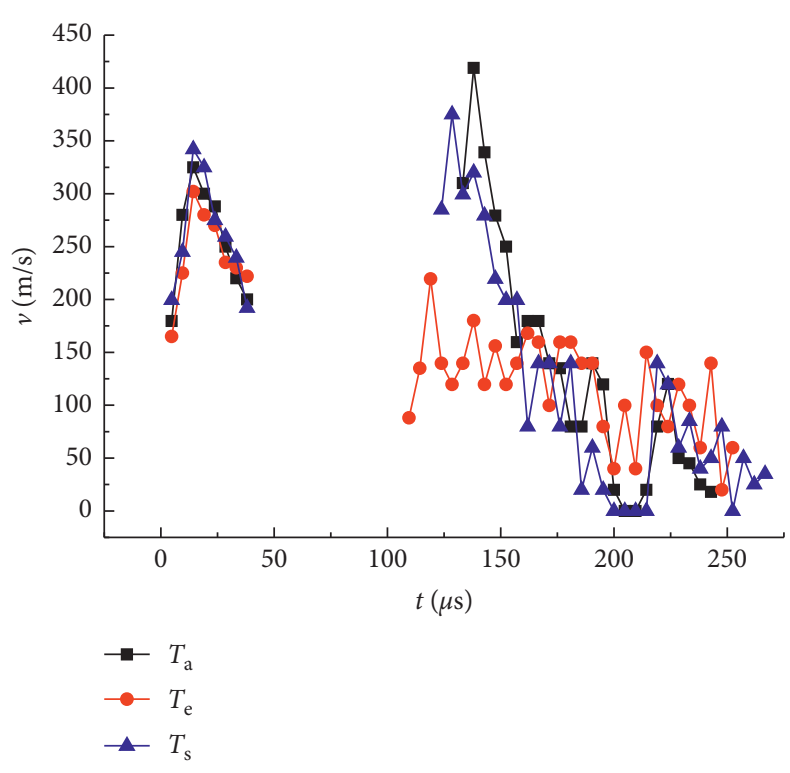

(b)

FIGURE 8: Changing curves of dynamic mechanical parameters of the running crack with time in the rectangular defect group. (a) $K_{\mathrm{I}}-t$. (b) $v-t$.

stage, the time initiating at the upper edge of the circular defect in the three specimens and their corresponding stress intensity factor are listed in Table 2.

Table 2 shows that the air-filled stress intensity factor is the largest, followed by silicone rubber-filled stress intensity factor, and then epoxy-filled stress intensity factor, indicating that the filling material has a significant effect on the initiation behavior of the running crack at the defect. After running crack initiation, the stress intensity factors rapidly reach the peak value, and the peak values of specimens $C_{\mathrm{a}}, C_{\mathrm{e}}$, and $C_{\mathrm{s}}$ are $2.28,1.39$, and $1.70 \mathrm{MN} \cdot \mathrm{m}^{-3 / 2}$, respectively. Thereafter, the stress intensity factor gradually reduces until the running crack propagates through the specimen. In stage III, the propagation velocity of the running crack shows a similar trend to that of the stress intensity factor. After running crack initiation, the propagation velocity shows an increasing trend first, followed by a decreasing trend. The peak values of the running crack propagation velocity in specimens $C_{\mathrm{a}}, C_{\mathrm{e}}$, and $C_{\mathrm{s}}$ are $688.2,520.0$, and $610.3 \mathrm{~m} / \mathrm{s}$, respectively.

5.2. Analysis of the Rectangular Defect Group. In stage III, two running cracks are generated from the rectangular defect in specimens $T_{\mathrm{a}}, T_{\mathrm{e}}$, and $T_{\mathrm{s}}$. Due to the symmetry of the stress states of the two running cracks, the propagation form and the path of the two running cracks are basically the same. Therefore, the dynamic behavior of the running crack
TABLE 3: The initiating time and corresponding stress intensity factor at the upper edge of the rectangular defect.

\begin{tabular}{lccc}
\hline Specimen & $T_{\mathrm{a}}$ & $T_{\mathrm{e}}$ & $T_{\mathrm{s}}$ \\
\hline Initiating time $(\mu \mathrm{s})$ & 128.6 & 104.8 & 119.0 \\
Stress intensity factor $\left(\mathrm{MN} \cdot \mathrm{m}^{-3 / 2}\right)$ & 0.92 & 0.82 & 0.88 \\
\hline
\end{tabular}

initiated at the upper right corner of the rectangular defect in each specimen was analyzed, as shown below.

Figure 8 shows the changing curves of the stress intensity factor $K_{\mathrm{I}}$ and propagation velocity $v$ of the running crack with time in the rectangular defect group. In stage I, when initiating at the end of precrack, the stress intensity factor of the running cracks in specimens $T_{\mathrm{a}}, T_{\mathrm{e}}$, and $T_{\mathrm{s}}$ is $0.82,0.83$, and $0.85 \mathrm{MN} \cdot \mathrm{m}^{-3 / 2}$, respectively, which are basically the same as those of the circular defect group. In addition, the stress intensity factor and propagation velocity also show an increasing trend first, followed by a decreasing trend after the running crack initiation. The defect shape and filling material have no significant effect on the initiation and propagation behavior of the running crack in stage I.

In stage III, the time initiating at the upper edge of the rectangular defect in the three specimens and their corresponding stress intensity factor are shown in Table 3.

Table 3 shows that the air-filled stress intensity factor is the largest, followed by silicone rubber-filled stress intensity factor, and then epoxy-filled stress intensity factor. Since the 
two running cracks generated from the defect have a competitive relationship in the propagation process, the stress intensity factor of the running crack shows an oscillatory change during propagation. In stage III, the propagation velocity of the running crack also shows an oscillatory change, and the peak values of the running crack propagation velocity in the specimens are 419.0, 219.5, and $375.2 \mathrm{~m} / \mathrm{s}$.

The peak values of the stress intensity factor and propagation velocity of the running cracks in the rectangular defect group are significantly smaller than those in the circular defect group. Circular defect and rectangular defect have different effects on the propagation behavior of running crack in stage III.

\section{Conclusions}

In conclusion, the effect of defect shape (circular and rectangular) and filling material (air, epoxy, and silicone rubber) on the propagation behavior of the running crack was successfully studied by model experiments. The experiments show that the process of interaction between the defect and running crack can be divided into three stages.

In stage I, defect shape and filling materials have no significant effect on the initiation and propagation behavior of the running crack. The stress intensity factor of the running crack when initiating at the end of precrack is approximately $0.85 \mathrm{MN} \cdot \mathrm{m}^{-3 / 2}$.

In stage II, both defect shape and filling material have a significant effect on the energy accumulation time of the running crack at the defect. In terms of the effect degree of the defect shape on the energy accumulation time, circular defect > rectangular defect; in terms of the effect degree of filling material on energy accumulation time, air $>$ silicone rubber > epoxy.

In stage III, both defect shape and filling material have a significant effect on the initiation and propagation behavior of the running crack. For circular defect, after the running crack initiation, both the stress intensity factor and propagation velocity increase first and then decrease. For rectangular defect, since the two running cracks generated from the defect have a competitive relationship in the propagation process, the stress intensity factor of the running crack shows an oscillatory change during propagation. The effect degree of defect shape on the stress intensity factor when initiating at the defect is in the following order: circular defect > rectangular defect; the effect degree of filling material when initiating at the defect is in the following order: air $>$ silicone rubber $>$ epoxy. This observation is consistent with the effect of defect shape and filling material on the accumulation time as mentioned above.

\section{Nomenclature}

$\mathrm{C}_{\mathrm{a}}, \mathrm{C}_{\mathrm{e}}$ and PMMA specimen containing circular defect

$\mathrm{C}_{s}$ : $\quad$ with filling material of air, epoxy and silicone rubber, respectively

c: $\quad$ Optical stress constant of specimen material

D: $\quad$ Diameter of caustics at mode I crack tip

$d: \quad$ Thickness of specimen
$F(v): \quad$ Correlation factor associated with the crack propagation velocity

g: $\quad$ Numerical factor

$K_{\mathrm{I}}: \quad \quad \quad$ Mode I stress intensity factor of the crack tip

$K_{\text {II }}: \quad$ Mode II stress intensity factor of the crack tip

PMMA: Polymethyl methacrylate

$R$ : $\quad$ Polar radius

$T_{\mathrm{a}}, T_{\mathrm{e}}$, and PMMA specimen containing rectangular defect

$T_{\mathrm{s}}$ : $\quad$ with filling material of air, epoxy, and silicone rubber, respectively

$t: \quad$ Time

$v: \quad$ Instantaneous velocity of crack propagation

$x^{\prime}, y^{\prime}: \quad$ Abscissa and ordinate of caustics on the reference plane

$z_{0}$ : $\quad$ Distance between the specimen and the reference plane

$\Delta s: \quad$ Crack tip displacement difference of two successive caustics photos

$\Delta t: \quad$ Time interval between two successive caustics photos

$\theta: \quad$ Polar radius.

\section{Data Availability}

The data used to support the findings of this study are available from the corresponding author upon request.

\section{Conflicts of Interest}

The authors declare no conflicts of interest regarding the publication of this article.

\section{Acknowledgments}

This research was supported by the National Natural Science Foundation of China (no. 51664007).

\section{References}

[1] B. Dai, G. C. He, and Z. J. Zhang, "A numerical research on crack process of gypsum containing single flaw with different angle and length in uniaxial loading," Shock and Vibration, vol. 2018, Article ID 2968205, 16 pages, 2018.

[2] B. Wasiluk and K. Golos, "Prediction of crack growth direction under plane stress for mixed-mode I and II loading," Fatigue Fracture of Engineering Materials and Structures, vol. 23, no. 5, pp. 381-386, 2000.

[3] M. R. M. Aliha, M. R. Ayatollahi, and R. Pakzad, "Brittle fracture analysis using a ring-shape specimen containing two angled cracks," International Journal of Fracture, vol. 153, no. 1, pp. 63-68, 2008.

[4] Y. Zhang, Z. N. Jiang, and J. Zhao, "Elasto-plastic dynamic behaviour of a free-free beam with an initial notch," Journal of Vibration and Shock, vol. 26, no. 10, pp. 27-29, 2007.

[5] J. S. Hu, X. Feng, and J. Zhou, "Vibration analysis and crack identification of a cantilever pipe with a circumferential partthrough crack," Journal of Vibration and Shock, vol. 30, no. 4, pp. 21-25, 2011.

[6] P. P. Cortet, G. Huillard, and V. S. Ciliberto, "Attractive and repulsive cracks in a heterogeneous material," Journal of 
Statistical Mechanics Theory \& Experiment, vol. 30, no. 2, pp. 155-168, 2008.

[7] L. Marsavina, E. Linul, T. Voiconi, and T. Sadowski, "A comparison between dynamic and static fracture toughness of polyurethane foams," Polymer Testing, vol. 32, no. 4, pp. 673-680, 2013.

[8] J. Koivisto, M. J. Dalbe, M. J. Alava, and S. Santucci, "Path (un) predictability of two interacting cracks in polycarbonate sheets using digital image correlation," Scientific Reports, vol. 6, no. 1, Article ID 32278, 2016.

[9] M. L. Fender, F. Lechenault, and K. E. Daniels, "Universal shapes formed by two interacting cracks," Physical Review Letters, vol. 105, no. 12, pp. 2058-2064, 2010.

[10] R. S. Yang, C. X. Ding, L. Y. Yang, P. Xu, and C. Chen, "Hole defects affect the dynamic fracture behavior of nearby running cracks," Shock and Vibration, vol. 2018, Article ID 5894356, 8 pages, 2018.

[11] Z. W. Yue, Y. Song, R. J. Han, W. Zhang, and C. Guo, "Experimental study on dynamic fracture behavior of threepoint bending beam with double deformity inclusions," Journal of Southeast University (English Edition), vol. 32, no. 3, pp. 333-338, 2016.

[12] E. Linul, N. Movahedi, and L. Marsavina, "The temperature and anisotropy effect on compressive behavior of cylindrical closed-cell aluminum-alloy foams," Journal of Alloys and Compounds, vol. 740, pp. 1172-1179, 2018.

[13] M. Li, Z. M. Zhu, R. F. Liu, B. Liu, L. Zhou, and Y. Q. Dong, "Study of the effect of empty holes on propagating cracks under blasting loads," International Journal of Rock Mechanics and Mining Sciences, vol. 103, pp. 186-194, 2018.

[14] M. R. M. Aliha, G. R. Hosseinpour, and M. R. Ayatollahi, "Application of cracked triangular specimen subjected to three-point bending for investigating fracture behavior of rock materials," Rock Mechanics and Rock Engineering, vol. 46, no. 5, pp. 1023-1034, 2013.

[15] Q. Zhou, Z. M. Zhu, X. Wang, J. Zhou, L. Lang, and X. Zhang, "The effect of a pre-existing crack on a running crack in brittle material under dynamic loads," Fatigue \& Fracture of Engineering Materials \& Structures, vol. 42, no. 11, pp. 2544-2557, 2019.

[16] Z. J. Wu and L. N. Y. Wong, "Investigating the effects of micro-defects on the dynamic properties of rock using numerical manifold method," Construction \& Building Materials, vol. 72, pp. 72-82, 2014.

[17] L. Marsavina, T. Sadowski, and M. Knec, "Crack propagation paths in four point bend Aluminium-PMMA specimens," Engineering Fracture Mechanics, vol. 108, pp. 139-151, 2013.

[18] F. Berto and M. R. Ayatollahi, "Fracture assessment of Brazilian disc specimens weakened by blunt V-notches under mixed mode loading by means of local energy," Materials and Design, vol. 32, no. 5, pp. 2858-2869, 2011.

[19] Y. J. Chao, S. Liu, and B. J. Broviak, "Brittle fracture: variation of fracture toughness with constraint and crack curving under mode I conditions," Experimental Mechanics, vol. 41, no. 3, pp. 232-241, 2001.

[20] M. R. Ayatollahi and M. R. M. Aliha, "Analysis of a new specimen for mixed mode fracture tests on brittle materials," Engineering Fracture Mechanics, vol. 76, no. 11, pp. 15631573, 2009.

[21] M. R. M. Aliha and M. R. Ayatollahi, "Geometry effects on fracture behaviour of polymethyl methacrylate," Materials Science and Engineering A, vol. 527, no. 3, pp. 526-530, 2010.

[22] H. Saghafi, A. Zucchelli, and G. Minak, "Evaluating fracture behavior of brittle polymeric materials using an IASCB specimen," Polymer Testing, vol. 32, no. 1, pp. 133-140, 2013.
[23] L. Y. Yang, R. S. Yang, and P. Xu, "Caustics method combined with laser and digital high-speed camera and its applications," Journal of China University of Mining and Technology, vol. 42, no. 2, pp. 188-194, 2013.

[24] R. S. Yang, C. X. Ding, L. Y. Yang, and C. Chen, "Model experiment on dynamic behavior of jointed rock mass under blasting at high-stress conditions," Tunnelling and Underground Space Technology, vol. 74, pp. 145-152, 2018.

[25] R. S. Yang, P. Xu, Z. W. Yue, and C. Chen, "Dynamic fracture analysis of crack-defect interaction for mode I running crack using digital dynamic caustics method," Engineering Fracture Mechanics, vol. 161, pp. 63-75, 2016. 